\title{
Effect of N-Acetyl-D-Glucosamine on Gene Expression in Vibrio parahaemolyticus
}

\author{
Fabiano L. Thompson ${ }^{1}$, Antonio Alves Neto ${ }^{1}$, Eidy de O. SAntos ${ }^{1}, \mathrm{KaORI}_{\text {IzUTSu }}^{2}$, and Tetsuya IidA ${ }^{2}$ \\ ${ }^{1}$ Department of Genetics, Institute of Biology, Federal University of Rio de Janeiro (UFRJ) Brazil; and ${ }^{2}$ Laboratory of \\ Genomic Research on Pathogenic Bacteria, International Research Center for Infectious Diseases, Research Institute \\ for Microbial Diseases, Osaka University, Suita, Osaka 565-0871, Japan
}

(Received July 29, 2010—Accepted November 6, 2010—Published online December 14, 2010)

We analyzed the effect of N-acetyl-D-glucosamine (GlcNAc) on gene expression in the marine bacterium Vibrio parahaemolyticus. The total number of genes whose expression was induced and repressed genes in the presence of GlcNAc was 81 and 55, respectively. The induced genes encoded a variety of products, including proteins related to energy metabolism (e.g. GlcNAc and chitin utilization), transport, central metabolism and chemotaxis, hypothetical proteins, mannose-sensitive hemagglutinin pilus (MSHA), and a PilA protein, whereas the repressed genes encoded mainly hypothetical proteins. GlcNAc appears to influence directly or indirectly a variety of cellular processes, including energy metabolism, chitin utilization, competence, biofilm formation and pathogenicity. GlcNAc, one of the most abundant aminosugars in the oceans, is used by $V$. parahaemolyticus as an energy source and affects the cellular functioning of this marine bacterium.

Key words: vibrios, V. parahaemolyticus, N-acetyl-D-glucosamine, microarrays, genome plasticity

N-acetyl-D-glucosamine (GlcNAc), the building block of the chitin polymer, is one of the most abundant aminosugars in the oceans $(11,20)$. Marine organisms, particularly zooplankton, produce significant quantities of chitin in the form of carapaces. Chitin is insoluble and made available as a nutrient to fuel the marine microbial food web by means of chitinolytic microrganisms that transform it into its building blocks $(4,18,38,45)$. Chitin and GlcNAc appear to be important sources of carbon and nitrogen for vibrios $(2,3$, $22,24)$. These bacteria seem to be major players in the cycling of chitin in the marine environment as they produce a variety of potent chitinolytic enzymes $(3,30,40)$. GlcNAc uptake and metabolism is mediated by the nag regulon which comprises four main genes $(\operatorname{nag} A B C E)(6,9,19,20)$. The transport of GlcNAc into the cell is mediated by a specific PTS (phosphotransferase system) enzyme encoded by nagE. This enzyme transforms the aminosugar in GlcNAc-6-P and pyruvate. Within the cell, GlcNAc-6-P is processed by at least six enzymes and the end products are fructose-6-P, $\mathrm{NH}_{3}$ and acetate. GlcNAc-6-P is transformed in glucosamine-6-P (GlcN-6-P) by N-acetyl-D-glucosamine deacetylase encoded by the $n a g A$ gene). Subsequently, GlcN-6-P is transformed in fructose-6-P (or used in the biosynthesis of the cell envelope path) by glucosamine-6-P deaminase (isomerase; encoded by the $\operatorname{nag} B$ gene). Fructose-6-P can be used in glycolysis to generate energy or it can be processed by glucosamine-6-P synthase, again generating GlcN-6-P. In Vibrio cholerae, the gene $n a g B$ is on chromosome II, whereas nagE, nag $A$ and nag $C$ are on chromosome I (14). Some $V$. cholerae strains have a homologous copy of nagA in the vibrio pathogenicity island 2 (17).

The expression of nag genes in Escherichia coli is induced

\footnotetext{
* Corresponding author. E-mail: fabiano.thompson@biologia.ufrj.br; Tel: +55-21-25626567; Fax: +55-21-25626333.
}

in the presence of GlcNAc and tightly regulated by the cAMP-CRP (cyclic AMP receptor protein) complex $(32,33$, $36,37)$. In $E$. coli, $\mathrm{NagC}$ is a repressor of $n a g A B E(31,34$, $35)$ It binds to two sites in the intergenic regions between $n a g B$ and $n a g E$. In addition, there is a cAMP-CRP binding site between the two NagC-binding sites. The two binding sites, named boxes $\mathrm{E}$ and $\mathrm{B}$, are oriented to control the expression from the promoters of $n a g E$ and $n a g B$, respectively. In the absence of sugars, $\mathrm{NagC}$ represses gene expression and activates the expression of $g \operatorname{lm} U S$. This operon encodes the enzymes that form GlcNAc-6-P and UDPGlcNAc. In $V$. cholerae the two binding sites for NagC and the cAMP-CRP binding site are in the intergenic regions of $n a g E$ and $n a g A C$ (44). The expression of $n a g B$ is induced in the presence of GlcNAc, but not by glycerol, D-glucosamine, or D-glucose. It appears that the $\operatorname{nag} B$ promoter is not affected by D-glucose catabolic repression and is induced even in the presence of glucose and GlcNAc. In order to understand the effect of GlcNAc in different cellular processes of vibrios, a comprehensive approach is needed to permit the concomitant examination of different operons and regulons.

Whole genome sequencing has enabled the construction of microarrays which contain all genes of a given bacterium in a single chip. This technology provides a comprehensive view of global bacterial gene expression. A series of striking studies using microassays demonstrated that chitin (but not GlcNAc) induced competence and serogroup conversion in $V$. cholerae $(4,5,26,27)$. A total of 22 induced genes and 51 repressed genes were found in Vibrio cholerae grown on GlcNAc $(26,27)$. The induced genes included those encoding for the nagEBA-1 operon (PTS transport system), the GlcNAc-binding protein VCA0811, and the chemotaxis protein VC0449. The repressed genes encoded succinyl-CoA synthase, components of 2-oxoglutarate dehydrogenase, phos- 
phoenolpyruvate carboxylase, NadC transporter, D-glucose binding protein of the ABC transporter, and glyceraldehyde3-P dehydrogenase. Most of the information concerning chitin and GlcNAc utilization and its effect on gene expression is restricted to a few vibrio species, mainly $V$. cholerae. More than one hundred and fifteen vibrio species have been described, yet it is not known if GlcNAc also influences the processes of chemotaxis, adherence, and competence in other vibrios $(2,3,6,9,13,19,20)$. The aim of the present study was to determine the gene expression profile of Vibrio parahaemolyticus raised on GlcNAc in different stages of the growth curve by means of a microarray analysis.

\section{Materials and Methods}

\section{Bacterial strain and growth conditions}

Vibrio parahaemolyticus RIMD2210633 was originally isolated from a patient suffering from diarrhea in the quarantine sector of Kansai International Airport in 1996. This strain harbors the $t d h$ gene and belongs to the O3:K6 serogroup. It has been detected in both clinical cases and in the marine environment (41). RIMD2210633 was grown in the minimum medium M9 (Difco ${ }^{\mathrm{TM}}$, Sparks, USA) supplemented with $0.5 \%$ maltose and $3 \% \mathrm{NaCl}$ for 8 hours at $28^{\circ} \mathrm{C}$ prior to the experiments. The experiments comprised two treatments (maltose and maltose+GlcNAc) with 2 replicates each. An innoculum of the bacterial culture $(5 \mathrm{~mL})$ was added into an Erlenmeyer flask $(1 \mathrm{~L})$ containing $500 \mathrm{~mL}$ of $\mathrm{M} 9+3 \% \mathrm{NaCl}$ supplemented with $0.5 \%$ de maltose (Sigma-Aldrich, St. Louis, USA) or $0.5 \%$ maltose $+0.5 \%$ GlcNAc (Sigma-Aldrich). The flasks were kept in aerobic conditions with gentle shaking at $28^{\circ} \mathrm{C}$ for up to 72 hours. Aliquots of the bacterial growth culture were taken from the replicates at 24,44 and 72 hours for the microarray gene expression analysis. The microarray experiments were performed essentially as described previously (16). Bacterial growth was assessed by measuring optical density at $550 \mathrm{~nm}$.

\section{RNA extraction and $c D N A$ preparation}

Aliquots $(50 \mathrm{~mL})$ of bacterial culture were pelleted and resuspended in $5 \mathrm{~mL}$ of TRIZOL (Invitrogen, Carlbad, USA). Nucleic acids were extracted using $2 \mathrm{~mL}$ of chloroform (Merck KGaA, Darmstadt, Germany). Samples were incubated for 5 minutes at room temperature and centrifuged at $12000 \times g$ (gravity) for $20 \mathrm{~min}$ utes at $4^{\circ} \mathrm{C}$. Aliquots of $3 \mathrm{~mL}$ of the supernatant were placed in clean tubes containing $0.3 \mathrm{~mL}$ of sodium acetate $(3 \mathrm{M})$ and $3 \mathrm{~mL}$ of isopropyl alcohol (Merck). The tubes were incubated at $4{ }^{\circ} \mathrm{C}$ for 5 minutes to precipitate the nucleic acids and then centrifuged for 15 minutes at $12000 \times \mathrm{g}$ and $4^{\circ} \mathrm{C}$. In the final step, $2 \mathrm{~mL}$ of $70 \%$ ethanol (v/v; Merck) was added in order to remove salts. The mixture of nucleic acids was precipitated and treated with DNase I (Invitrogen). Then, $10 \mu \mathrm{L}$ of DNase I was added to $70 \mu \mathrm{L}$ of the mixture of nucleic acids (Invitrogen). The mixture was incubated for 1 hour at $37^{\circ} \mathrm{C}$. A total of $80 \mu \mathrm{L}$ acidic phenol (Invitrogen; around $\mathrm{pH} 5$ ) and $80 \mu \mathrm{L}$ of chloroform (Merck) were added and the mixture was vortexed for 5 minutes. The mixture was placed in a clean container containing $16 \mu \mathrm{L}$ of sodium acetate $(3 \mathrm{M})$ and 400 $\mu \mathrm{L}$ of $100 \%$ ethanol $(\mathrm{v} / \mathrm{v})$ and kept on ice for 5 minutes. It was centrifuged for 15 minutes at $12000 \times g$ and $4^{\circ} \mathrm{C}$. The supernatant was discarded and $500 \mu \mathrm{L}$ of $70 \%$ ethanol $(\mathrm{v} / \mathrm{v})$ was added to wash the RNA pellet. Total RNA was purified using the RNeasy kit (Qiagen, Hilden, Germany). RNA tags were purified using UTPs labeled with an aminoallyl reactive group. This group binds to the dyes $\mathrm{Cy} 3$ and $\mathrm{Cy} 5$ used to label the samples. cDNA was prepared by mixing $20 \mu \mathrm{g}$ purified total RNA, $2 \mu \mathrm{L}$ of random primers (Invitrogen) and $22 \mu \mathrm{L}$ of RNase-free water. This mixture was incubated for 5 minutes at $70^{\circ} \mathrm{C}$ and placed on ice. In the next stage $8.0 \mu \mathrm{L}$ of first-strand buffer (Invitrogen), $2.0 \mu \mathrm{L}$ of DDT (dithiothreitol; GE Healthcare Biosciences AB, Uppsala, Sweden)
$(0.1 \mathrm{M}), 4.0 \mu \mathrm{L}$ of dNTP-aminoallyldUTP and $4.0 \mu \mathrm{L}$ of reverse transcriptase SuperScriptIII (Invitrogen) were added and the mixture was incubated for 3 hours at $46^{\circ} \mathrm{C}$ in order to generate a sufficient amount of cDNA. A total of $60 \mu \mathrm{L}$ of $\mathrm{H}_{2} \mathrm{O}$ and $100 \mu \mathrm{L}$ of phenol-chloroform (Invitrogen) were added and mixed in vigorously. After centrifugation of this mixture of cDNA for 5 minutes at $12000 \times g$, the supernatant was removed and added to a container containing $10 \mu \mathrm{L}$ of sodium acetate $(3 \mathrm{M})$ and $250 \mu \mathrm{L}$ of ethanol $(100 \%)$. The mixture was kept on ice for 5 minutes and centrifuged for 15 minutes at $12000 \times g$ and $4{ }^{\circ} \mathrm{C}$. The supernatant was removed carefully and $500 \mu \mathrm{L}$ of ethanol $(70 \%)$ was added to remove impurities and products not incorporated into the cDNA. The ethanol was removed and the sample was dried at room temperature for 5 minutes.

\section{Cy3 and Cy5 labeling of cDNA and microarray hybridization}

The resulting cDNA pellet was labeled with $\mathrm{Cy} 3$ or Cy5. To mark the total cDNA with the fluorescent dyes, the pellet was resuspended in $10 \mu \mathrm{L}$ of sodium bicarbonate $(50 \mathrm{mM}, \mathrm{pH} 9)$. Ten microliters of $\mathrm{Cy} 3$ was added to the sample of cDNA originating from the control (maltose) treatment and $10 \mu \mathrm{L}$ of $\mathrm{Cy} 5$ was added to the sample originating from the test treatment (maltose+GlcNAc). Each sample was incubated for 1 hour in the dark. Excess dye was removed with the aid of SEP columns (Applied Biosystems Life Technologies Carlsbad, USA). The dried labeled cDNA was dissolved in $20 \mu \mathrm{L}$ of water. The hybridization solution was prepared by mixing $20 \mu \mathrm{L}$ of cDNA dissolved with $3 \mu \mathrm{L}$ of sodium dodecyl sulfate $10 \%$ (Sigma-Aldrich), $6 \mu \mathrm{L}$ of yeast tRNA (Invitrogen) and $15 \mu \mathrm{L}$ of SSC (20×, $3 \mathrm{M} \mathrm{NaCl}, 0.3 \mathrm{M}$ citrate sodium, pH 7.0). This mixture was incubated for 5 minutes at $96^{\circ} \mathrm{C}$, and then immediately incubated for 5 minutes at $60^{\circ} \mathrm{C}$. Aliquots of labeled cDNA $(10 \mu \mathrm{L})$ of each sample (control and test) were mixed and placed on a microarray slide, because the experiments are based on competitive hybridization. The slide contains 4832 ORFs of $V$. parahaemolyticus. The microarray slides were simultaneously prepared to receive the mixture. First, the slides were washed with blocking solution $(7.5 \mathrm{~g}$ of succinic anhydride, $472.5 \mathrm{~mL}$ of 1-Methy-2-pyrrolidinone and $1.0 \mathrm{M}$ sodium borate $\mathrm{pH} 8.0$ ). Then the slides were washed with water twice for 10 minutes. The slides were washed with absolute ethanol and dried in a hybridization apparatus (MAUI BioMicro Lid Systems, BioMicro, Salt Lake City, USA). The mixtures containing cDNA were added to the slides and kept in the hybridization apparatus for 16 hours at $60^{\circ} \mathrm{C}$. The slides were washed with SSC buffer three consecutive times and once with absolute ethanol.

\section{Microarray analysis}

After washing and drying, the slides were examined using a scanner, the ScanArray Lite Express (Perkin Elmer Life Sciences, Massachusetts, USA), that enables the detection and quantification of fluorescence. To determine the expression of each gene, $\log 2$ values of fluorescence for the maltose+GlcNAc treatment were divided by those for the control treatment (maltose) for each spot. Gene expression was considered induced or repressed if the fluorescence values were $>2$ or $<-2$, respectively, representing a four-fold increase or decrease in fluorescence compared with the control. Dendrograms were constructed using MatLab 7.2.0 on the basis of Euclidian Distance similarity matrices and the Average Linkage clustering method. The localization of the genes in the metabolic pathways was performed using Kyoto Encyclopedia of Genes and Genomes (KEGG).

\section{Results}

V. parahaemolyticus RIMD2210633 grown with maltose+ GlcNAc showed a biphasic growth curve with two exponential phases (i.e. 1-11 hours and 22-27 hours) and two stationary phases (i.e. aprox. 12-21 hours and 44-72 hours), whereas the control treatment (maltose) resulted in only one exponential phase (i.e. 1-11 hours) and one stationary phase (i.e. 12-72 hours) (Fig. 1). 


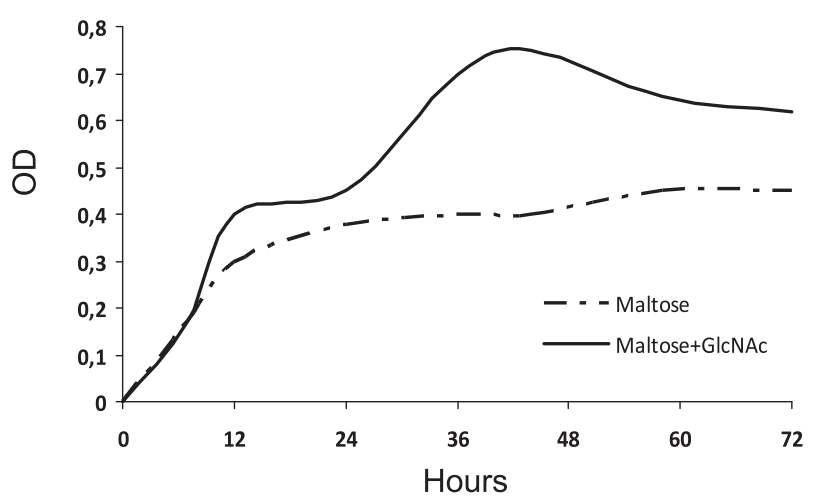

Fig. 1. Growth of $V$. parahaemolyticus on maltose and maltose+ GlcNAc during 72 hours.

The second exponential phase (22-27 hours) in the maltose+ GlcNAc treatment corresponded to the beginning of GlcNAc's utilization by the bacterium. To evaluate the gene expression induced and repressed by GlcNAc, we selected the time points 24 , 44 , and 72 hours to perform the microarray analysis. Overall, the induced and repressed genes were distributed in three main groups (Figs. 2 and 3). Group one contained genes induced or repressed towards the end of the experiment, between the 44 and 72 hour marks. Group two contained genes induced or repressed mainly in the initial 24 hours of experiment. Group 3 contained genes induced or repressed throughout the 72 hours of the experiment. A total of 81 genes had their expression induced during the experiment; 59 genes at 24 hours, 37 genes at 44 hours, and 31 genes at 72 hours. A total of 39 genes in chromosome I and 20 genes in chromosome II were induced to express by GlcNAc at 24 hours (Table S1). The induced genes encoded mainly proteins related to chemotaxis, substrate binding/transport, GlcNAc metabolism, energy metabolism, unknown processes (hypothetical proteins), and competence/chitin surface colonization (PilA protein). The genes VP0828, VP0829, VP0831, and VPA0038 were associated with the uptake and metabolism of GlcNAc. These genes were expressed throughout the experiment (Fig. 2). VPA0038 encodes NagB which transform GlcN-6P in Fru-6P (fructose-6-phosphate) and $\mathrm{NH}_{3}$. Fru-6-P subsequently enters the glycolysis pathway. A NagC repressor (VP0828) and a hypothetical protein (VP0830) inside the nag operon were induced too. Overall, genes related to chemotaxis, substrate binding/transport, and GlcNAc metabolism predominated.

A group of 55 genes had their expression repressed during the experiment; 38 genes at 24 hours, 24 genes at 44 hours, and 21 genes at 72 hours (Fig. 3). The genes repressed at 24 hours (26 genes in chromosome I and 12 genes in chromosome II) mainly encoded hypothetical proteins (e.g. VP0711, VP2388, VP2400, VP2487, and VPA0055) (Table S2). A putative operon comprising VP0583 and VP0584 was repressed in the presence of GlcNAc. VP0584 (an Isocitrate lyase) is responsible for the transformation of isocitrate in succinate and glyoxylate at a bifurcating point of the Krebs cycle (TCA). This point (glyoxylate pathway) is a bypass of two stages of the TCA, converting isocitrate in succinyl-CoA. Several genes involved in the degradation of starch (VPA0461), maltose (VPA1619), and glycogen (VPA1638 and VPA1641) were also repressed in the presence of GlcNAc throughout the experiment (Fig. 3).

\section{Discussion}

Chitin influences the growth and physiology of vibrios in the environment. Chitin may support a significant portion of marine bacterial productivity. The omnipresence of chitin in the marine environment might explain the widespread distribution of vibrios $(22,38)$. In the present study it was showed that chitin monomers (GlcNAc) also have a significant effect on the growth and physiology of $V$. parahaemolyticus as they induced or repressed genes associated with different cellular processes. For instance, a chitinase (VPA0055) was induced after 24 hours. However, there was no chitin in the medium. We hypothesize that the
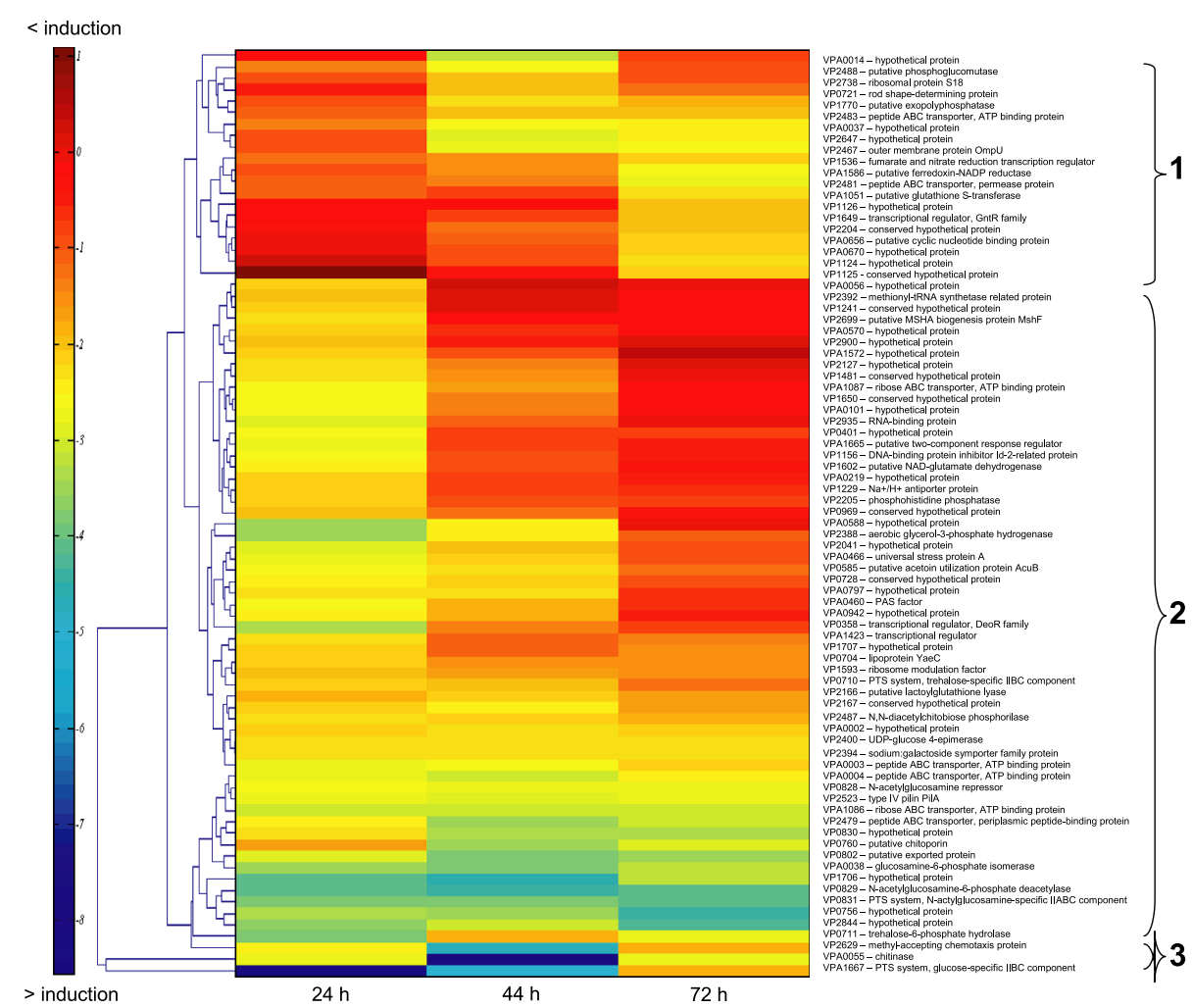

Fig. 2. Induced genes during the experiment. The genes induced to express at 24 , 44, and 72 hours are represented by three columns. The legend on the left indicates the level of induction. The gene number and product are shown on the right. The cluster was constructed using Euclidian distance similarity matrices and the Average Linkage clustering method. Highly induced genes appear in blue. Numbers on the right correspond to the three main groups of induced genes. 


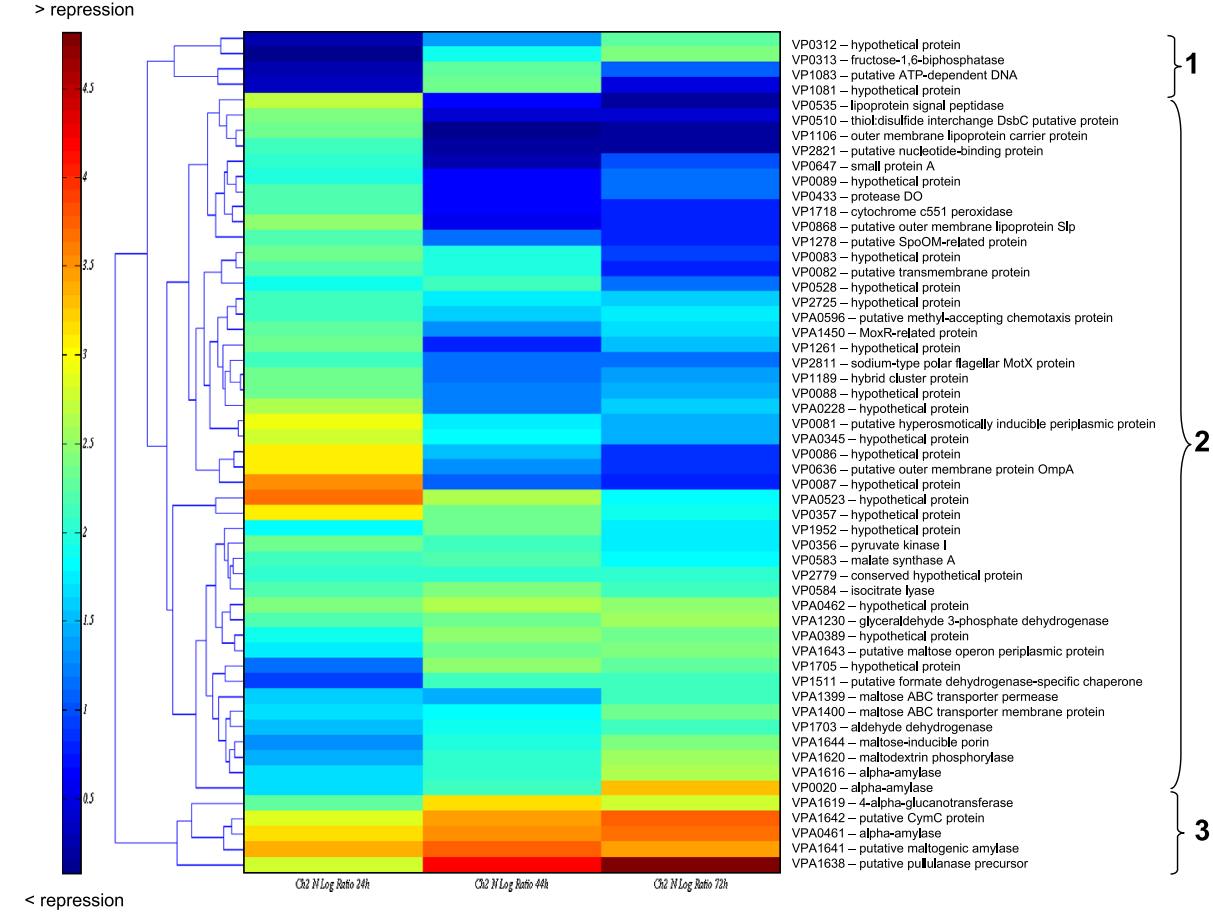

Fig. 3. Repressed genes during the experiment. The genes whose expression was repressed at 24,44 , and 72 hours are represented by three columns. The legend on the left indicates the level of repression. The gene number and product are shown on the right. The cluster was constructed using Euclidian distance similarity matrices and the Average Linkage clustering method. Highly repressed genes appear in brownish red. Numbers on the right correspond the three main groups of repressed genes. presence of GlcNAc in the bacterial cell might function as a signal to the bacterium, indicating the need to be prepared for a possible chitin-rich environment. VP2487 encodes a N,Ndiacetylchitobiose phosphorylase, a novel enzyme described in $V$. furnissii which promotes the reaction of chitobiose $\left(\mathrm{GlcNAc}_{2}\right)$ in NAG-1P and GlcNAc $(30,37)$. In $V$. cholerae, GlcNAc induces the expression of the GlcNAc-binding protein VCA0811, and a chemotaxis protein (VC0449). These facts further support our hypothesis. We suggest that the genes VP2487, VPA0055 and VP2629 belong to the nag regulon in $V$. parhaemolyticus. Because the gene organization and the response of the nag operon to GlcNAc in $V$. parahaemolyticus and $V$. cholerae is similar, we consider that the overall nag regulon was similar and $\mathrm{NagC}$ repressor dependent.

Potential operons (e.g. the operon composed of VP0710 and VP0711; that composed of VPA1086 and VPA1087; and that composed of VPA002, VPA0004, and VPA0003) were induced during the experiment. VP0710 encodes a membrane protein IIBC specific for threhalose (treP) of the PTS system. It transports threhalose into the cell and phosphorylates it. The intracellular threhalose-6-P is processed by threhalose-6-P hydrolase (VP0711, TreC) to produce glucose-6-P. In E. coli and B. subtilis, tre C and treP are organized in an operon induced by threhalose 6-P and negatively regulated by catabolic repression and by the repressor TreR $(1,8)$. The shortage of glucose in the medium might have been a signal for derepression. Another potential operon (VPA1086 and VPA1087) encodes the proteins ribose $\mathrm{ABC}$ transport (RbsA) and a ATP-binding protein (RbsD), respectively, which are responsible for the assimilation of R-ribose into the cell. VPA0003 (OppF) and VPA0004 (OppD) may form an operon responsible for the transport of peptide and nickel into the cell, whereas VPA002 (hypothetic protein), VPA003 and VPA004 may compose an induced operon of unknown function.

\section{Repressed genes are involved in energy metabolism}

$V$. parahaemolyticus clearly represses the expression of a set of genes in response to GlcNAc. Some of these genes were repressed during the entire experiment. For instance, the Pululanase VPA1638 hydrolyses glycogen by breaking $\alpha-1,6$-glycosidic bonds. The linear malto-oligosaccharides made available are then hydrolysed in maltose and maltotriose by the maltogenic amylase (VPA1641) (28). The gene VPA1619 encodes a 4- $\alpha$-glucanotransferase which transforms maltose in $\alpha$-D-glucose. The repression of these genes indicates a change during growth. After utilizing maltose, the bacterium may have repressed the expression of these genes in order to enter a second exponential growth phase as revealed in previous studies of the utilization of sugar mixtures by $E$. coli $(7,24)$.

The expression of genes associated with mechanisms of protein folding and secretion was also repressed. The gene VP0510 encodes a DsbC protein which along with the protein DsbA has a key role in the bisulfet bonds of newly formed proteins in the periplasm (46). The gene VP0535 encodes a membrane lipoprotein peptidase involved in the recognition and removal of signal peptides of lipoproteins prior to export. The gene VP1106 encodes LolA, a protein involved in the localization and fixation of the most abundant lipoprotein (Lpp) of the outer membrane (25). The relative repression of protein folding and secretion-related genes may be due to the two different growth phases. With the maltose+GlcNAc treatment, the cells are preparing to enter a new exponential phase, whereas with the control (maltose) treatment they are in a stationary phase where secretion is supposed to be greater.

The presence of GlcNAc may have resulted in the repression of glycolysis-related genes (VP0356 and VPA1230). 
VP0356 encodes a pyruvate kinase I which transforms phosphoenol-pyruvate in pyruvate, whereas VPA1230 encodes a glyceraldehyde 3-P dehydrogenase (Gap) which transforms glyceraldeyde- $3 \mathrm{P}$ in glycerol- $1,3 \mathrm{P}_{2}$. The gap gene is induced in the presence of glucose in B. subtilis (42). It would be reasonable to assume that glucose was not available in the present experiment as no Gap induction was observed. Another important effect of GlcNAc on the metabolism of $V$. parahaemolyticus is on the TCA cycle. The repressed malate synthase gene (VP0583) codes for the enzyme that transforms glyoxylate and acetyl-CoA in malate and the isocitrate lyase gene (VP0584) codes for the enzyme responsible for the transformation of isocitrate in succinate, suggesting that the glyoxylate pathway was not active. The glyoxylate pathway allows the production of a molecule with four carbons using two molecules of acetyl-CoA, spending only one $\mathrm{CO}_{2}$ molecule $(23,29)$. The activity of the isocitrate lyase directs the TCA cycle towards a more efficient biochemical path for bacterial growth. The glyoxylate pathway is repressed during cultivation in glucose and is induced in cultures with acetate in $E$. coli $(12,28)$. If we asssume that there is a high intracellular content of acetate in the maltose+GlcNAc treatment, E. coli and V. parahaemolyticus respond differently to similar conditions. This may also suggest that the TCA regulation in $V$. parahaemolyticus is different. Here we assume that there is a shortage of glucose but still a low intracellular level of acetate in the maltose +GlcNAc treated cells at 24 hours and $V$. parahaemolyticus adapts from glucose to GlcNAc utilization.

Two hypothetic operons (VP0081, VP0082, and VP0083; VP0086, VP0087, VP0088, and VP0089) were repressed in the presence of GlcNAc. The response of these genes in the experiments indicates their possible roles in $V$. parahaemolyticus cell physiology.

Induction by GlcNAc may promote horizontal gene transfer (via competence) and the expression of pathogenicity genes in $\mathrm{V}$. parahaemolyticus

A type IV pilin used to produce a type IV pilus was detected in this study. Competence is induced in $V$. cholerae by chitin. The pilus IV complex, DNA-binding proteins, and three regulatory cascades which are affected by the environment and cell physiology, are necessary in order for competence to take place. However, GlcNAc is not able to induce this mechanism in $V$. cholerae (26). A similar process could take place in $V$. parahaemolyticus but in response to GlcNAc. Another induced gene was VP2699, encoding a mannose-sensitive hemagglutinin pilus (MSHA) which is part of the biogenesis of the type IV pilus. MSHA stimulates adherence of $V$. cholerae on various surfaces (e.g. chitinous surfaces, intestinal mucus and abiotic surfaces), and is a key element in biofilm production $V$. cholerae (43). This gene contributes to the formation of a biofilm in $V$. parahaemolyticus (15). Several genes encoding structural and biosynthetic components related to MSH-pilus (24 hours: VP2693 putative MshP protein, VP2694 putative Type IV prepilin, MshO VP2698 putative V10 pilin, VP2699 putative MSHA biogenesis protein MshF, VP2705 MSHA biogenesis protein MshK, VP2706 MSHA biogenesis protein MshJ, and VP2709 single-strand binding protein; 44 hours:
VP2692 hypothetical protein, VP2693 putative MshP protein, VP2694 putative Type IV prepilin MshO, and VP2709 Single-strand binding protein; 72 hours: VP2694 putative Type IV prepilin MshO, VP2698 putative V10 pilin, VP2706 MSHA biogenesis protein MshJ) were expressed slightly below the signal threshold (between -1.1 and -1.9 ), indicating the induction of several related genes. In addition, a pathogenicity-related gene (VP0704) was also expressed. VP0704 encodes MetQ (YaeC), an antigenic protein of $V$. vulnificus, activated via Toll receptors (21). The induction of competence genes may influence the clonality of vibrios $(10,39)$

The present study provides new information on the gene expression of $V$. parahaemolyticus grown on GlcNAc. This aminosugar promotes cell growth and influences the physiology of this Vibrio by interfering with the gene expression associated with different cellular processes, most notably the processes for scavenging energy and genome plasticity.

\section{Acknowledgements}

FLT thanks JSPS, GOHO life Science Foundation, FAPERJ, and $\mathrm{CNPq}$ for grants. AAN thanks CAPES for a graduate scholarship. EOS thanks CNPq for a posdoc scholarship.

\section{References}

1. Arguelles, J.C. 2000. Physiological roles of trehalose in bacteria and yeasts: A comparative analysis. Arch. Microbiol. 174:217-224.

2. Bassler, B.L., P.J. Gibbons, C. Yu, and S. Roseman. 1991. Chitin utilization by marine bacteria. Chemotaxis to chitin oligosaccharides by $V$. furnissii. J. Biol. Chem. 266:24268-24275.

3. Bassler, B.L., P.J. Gibbons, C. Yu, and S. Roseman. 1991. Chitin utilization by marine bacteria. Degradation and catabolism to chitin oligosaccharides by V. furnissii. J. Biol. Chem. 266:24276-24286.

4. Blokesch, M., and G.K. Schoolnik. 2007. Serogroup conversion of Vibrio cholerae in aquatic reservoirs. PLOS Pathogens 3:733-742.

5. Blokesch, M., and G.K. Schoolnik. 2008. The extracellular nuclease Dns and its role in natural transformation of Vibrio cholerae. J. Bacteriol. 190:7232-7240.

6. Bouma, C.L., and S. Roseman. 1996. Sugar transport by the marine chitinolytic bacterium $V$. furnissii: Molecular cloning and analysis of the glucose and $\mathrm{N}$-acetyglucosamine permeases. J. Biol. Chem. 271:33457-33467.

7. Brückner, R., and F. Titgemeyer. 2002. Carbon catabolite repression in bacteria: Choice of the carbon source and autoregulatory limitation of sugar utilization. FEMS Microbiol. Letters 209:141-148.

8. Burklen, L., F. Schock, and M.K. Dahl. 1988. Molecular analysis of the interaction between the Bacillus subtilis trehalose repressor TreR and the tre operator. Mol. Gen. Genet. 60:48-55.

9. Chitlaru, E., and S. Roseman. 1996. Molecular cloning and characterization of a novel betaN-acetyldglucosaminidase from $V$. furnissii. J. Biol. Chem. 271:33433-33439.

10. Chiura, H.X., N. Uchiyama, and K. Kogure. 2009. Broad-host range gene transporter particles produced by Alivibrio fischeri. Microbes Environ. 24:322-329.

11. Cottrell, M.T., J.A. Moore, and D.L. Kirchman. 1999. Chitinases from uncultured marine microorganisms. Appl. Environ. Microbiol. 65:2553-2557.

12. Cozzone, A.J. 1998. Regulation of acetate metabolism by protein phophorylation in enteric bacteria. Ann. Rev. Microbiol. 52:127-164.

13. Gulig, P.A., M.S. Tucker, P.C. Thiaville, J.L. Joseph, and R.N. Brown. 2009. USER friendly cloning coupled with chitin-based natural transformation enables rapid mutagenesis of Vibrio vulnificus. Appl. Environ. Microbiol. 75:4936-4949.

14. Heidelberg, J.F., J.A. Eisen, W.C. Nelson, et al. 2000. DNA sequence of both 58 chromosomes of the cholera pathogen Vibrio cholerae. Nature 406:477-483. 
15. Honda, T., Y. Ni, and T. Miwatani. 1988. Purification and characterization of a hemolysin produced by a clinical isolate of kanagawa phenomenon negative Vibrio parahaemolyticus and related to the thermostable direct hemolysin. Infect. Immun. 56:961-965.

16. Izutsu, K., K. Kurokawa, K. Tashiro, S. Kuhara, T. Hayashi, T. Honda, and T. Iida. 2008. Comparative genomic analysis using microarray demonstrates a strong correlation between the presence of the 80-kilobase pathogenicity island and pathogenicity in kanagawa phenomenon-positive Vibrio parahaemolyticus strains. Infect. Immun. 76:1016-1023.

17. Jermyn, W.S., and E.F. Boyd. 2002. Characterization of a novel Vibrio pathogenicity island (VPI-2) encoding neuraminidase (nanH) among toxigenic Vibrio cholerae isolates. Microbiology 148:36813693.

18. Kajiwara, H., M. Toda, T. Mine, H. Nakada, H. Wariishi, and T. Yamamoto. 2010. Visualization of sialic acid produced on bacterial cell surfaces by lectin staining. Microbes Environ. 25:152-155.

19. Keyhani, N.O., and S. Roseman. 1996. The chitin catabolic cascade in the marine bacterium Vibrio furnissii: Molecular cloning, isolation, and characterization of a periplasmic beta- $\mathrm{N}$-acetylglucosaminidase. J. Biol. Chem. 271:33425-33432.

20. Keyhani, N.O., and S. Roseman. 1999. Physiological aspects of chitin catabolism in marine bacteria. Biochem. Biophys. Acta. 1473:108-122.

21. Kim, Y.R., S.E. Lee, C.M. Kim, et al. 2003. Characterization and pathogenic significance of Vibrio vulnificus antigens preferentially expressed in septicemic patients. Infect. Immun. 71:5461-5471.

22. Kirchman, D.L., and J. White. 1999. Hydrolysis and mineralization of chitin in the Delaware estuary. Aquat. Microb. Ecol. 18:187-196.

23. Kornberg, H.L. 1966. The role and control of the glyoxylate cycle in Escherichia coli. Biochem. J. 99:1-11.

24. Lendenmann, U., M. Snozzi, and T. Egli. 1996. Kinetics of the simultaneous utilization of sugar mixtures by Escherichia coli in continuous culture. Appl. Environ. Microbiol. 6:1493-1499.

25. Matsuyama, S., N. Yokota, and H. Tokuda. 1997. A novel outer membrane lipoprotein, LolB (hemM), involved in the LolA (p20)dependent localization of lipoproteins to the outer membrane of Escherichia coli. EMBO J. 16:6947-6955.

26. Meibom, K.L., X.B. Li, A.T. Nielsen, C.Y. Wu, S. Roseman, and G.K. Schoolnik. 2004. The Vibrio cholerae chitin utilization program. Proc. Natl. Acad. Sci. USA 101:2524-2529.

27. Meibom, K.L., M. Blokesch, N.A. Dolganov, C. Wu, and G.K. Schoolnik. 2005. Chitin induces natural competence in Vibrio cholerae. Science 310:1824-1827.

28. Nimmo, H.G., A.C. Borthwick, E.M. El Mansi, W.H. Holms, C. MacKintosh, and G.A. Nimmo. 1987. Regulation of the enzymes at the branchpoint between the citric acid cycle and the glyoxylate bypass in Escherichia coli. Biochem. Soc. Symp. 54:93-101.

29. Ornston, L.N., and M.K. Ornston. 1969. Regulation of glyoxylate metabolism in Escherichia coli K-12. J. Bacteriol. 98:1098-1108.

30. Park, J.K., N.O. Keyhani, and S. Roseman. 2000. Chitin catabolism in the marine bacterium Vibrio furnissii: Identification, molecular cloning, and characterization of an N,N'-diacetylchitobiose phosphorylase. J. Biol. Chem. 275:33077-33083.

31. Plumbridge, J.A. 1989. Sequence of the nagBACD operon in Escherichia coli $\mathrm{K} 12$ and pattern of transcription within the nag regulon. Mol. Microbiol. 3:505-515.
32. Plumbridge, J.A. 1990. Induction of the nag regulon of Escherichia coli by $\mathrm{N}$-acetylglucosamine and glucosamine: Role of the cyclic AMP-catabolite activator protein complex in expression of the regulon. J. Bacteriol. 172:2728-2735.

33. Plumbridge, J. 1995. Co-ordinated regulation of amino sugar biosynthesis and degradation: The $\mathrm{NagC}$ repressor acts as both an activator and a repressor for the transcription of the glmUS operon and requires two separated NagC binding sites. EMBO J. 14:3958-3965.

34. Plumbridge, J. 1999. Expression of the phosphotransferase system both mediates and is mediated by Mlc regulation in Escherichia coli. Mol. Microbiol. 33:260-273.

35. Plumbridge, J. 2000. A mutation which affects both the specificity of PtsG sugar transport and the regulation of $p t s G$ expression by Mlc in Escherichia coli. Microbiology 146:2655-2663.

36. Plumbridge, J., and A. Kolb. 1991. CAP and Nag repressor binding to the regulatory regions of the $n a g E-B$ and manX genes of Escherichia coli. Mol. Microbiol. 217:661-679.

37. Plumbridge, J., and A. Kolb. 1993. DNA loop formation between Nag repressor molecules bound to its two operator sites is necessary for repression of the nag regulon of Escherichia coli in vivo. Mol. Microbiol. 10:973-981.

38. Riemann, L., and F. Azam. 2002. Widespread N-acetyl-Dglucosamine uptake among pelagic marine bacteria and its ecological implications. Appl. Environ. Microbiol. 68:5554-5562.

39. Sawabe, T., S. Koizumi, Y. Fukui, S. Nakagawa, E.P. Ivanova, K. Kita-Tsukamoto, K. Kogure and F.L. Thompson. 2009. Mutation is the main driving force in the diversification of the Vibrio splendidus clade. Microbes Environ. 24:281-285.

40. Svitil, A.L., S. Chadhain, J.A. Moore, and D.L. Kirchman. 1997. Chitin degradation proteins produced by the marine bacterium Vibrio harveyi growing on different forms of chitin. Appl. Environ. Microbiol. 63:408-413.

41. Thompson, F.L., I. Cleenwerck, J. Swings, J. Matsuyama, and T. Iida. 2007. Genomic diversity and homologous recombination in Vibrio parahaemolyticus as revealed by Amplified Fragment Length Polymorphism (AFLP) and Multilocus Sequence Analysis (MLSA). Microbes Environ. 22:373-379.

42. Tobisch, S., D. Zuhlke, J. Bernhardt, J. Stulke, and M. Hecker. 1999. Role of Ccpa in regulation of the central pathways of carbon catabolism in Bacillus subtilis. J. Bacteriol. 181:6996-7004.

43. Watnick, P.I., K.J. Fullner, and R. Kolter. 1999. A role for the mannose-sensitive hemagglutinin in biofilm formation by Vibrio cholerae El Tor. J. Bacteriol. 181:3606-3609.

44. Yamano, N., N. Oura, J. Wang, and S. Fujishima. 1997. Cloning and sequencing of the genes for $\mathrm{N}$-acetylglucosamine use that construct divergent operons (nagE-nagAC) from Vibrio cholerae non-O1. Biosc. Biotechnol. Biochem. 61:1349-1353.

45. Yu, C., A.M. Lee, B.L. Bassler, and S. Roseman. 1991. Chitin utilization by marine bacteria: A physiological function for bacterial adhesion to immobilized carbohydrates. J. Biol. Chem. 266:2426024267.

46. Zapun, A., D. Missiakas, S. Raina, and T.E. Creighton. 1995. Structural and functional characterization of Dsbc, a protein involved in disulfet bond formation in Escherichia coli. Biochemistry 34:50755089 . 